\title{
THEORY OF CRYSTAL STRUCTURE, WITH APPLICATION TO TWENTY CRYSTALS BELONGING TO THE CUBIC OR ISOMETRIC SYSTEM.
}

\author{
By Albert C. Crehore.
}

$\mathrm{N}$ a former communication ${ }^{1}$ an expression for the mechanical force between any two atoms in their most general positions was derived from the early form of electromagnetic equations proposed by Thomson. ${ }^{2}$ The assumption was made that these equations apply to the individual electrons in the atoms, each revolving in circular orbits around a common center determined by the positive charge, and that the atoms are neutral, the total positive charge being numerically equal to the sum of the negative charges. The total force between the atoms is obtained by the summation of the forces between their various parts. To obtain the forces between the two positive charges, and between the positive charge of the one and an electron in the other, presents little difficulty because the ordinary electrostatic forces apply in these cases. The problem then resolves itself into that of finding the average force between two electrons supposed to be revolving in circular orbits with uniform velocity, the circles being in their most general positions in space. The result for two atoms is given by equations (23)-(25), page 755 of the paper referred to.

More recently ${ }^{2}$ the same problem has been solved for two atoms using the Lorentz form of the electromagnetic equations, involving the conception of retarded potentials which does not form a part of the older Thomson equations. The expression for the average force is finally developed in both cases in the form of infinite series of the inverse powers of $r$, the distance between the centers of the two atoms. In the Lorentz form the series begins with the inverse first power of $r$ and all inverse powers of $r$ are present. When, however, the force is resolved along the line joining the centers of the two atoms this series begins with the inverse square term. If the distance between the atoms is large, the first term is the only one which is effective, and we have in this result the suggestion that this force may be identical with that of gravitation. A critical

${ }^{1}$ Phil. Mag., June, I915, p. 750.

${ }^{2}$ J. J. Thomson, Phil. Mag., April, I88r, p. 229.

${ }^{3}$ Phys. Rev., June, I9I 7 . 
examination has shown that this force resembles that of gravitation in many ways, in being always an attraction and never a repulsion, and in being independent of the orientation of the axes of rotation of the atoms, so that two crystals have the same pull no matter how they are oriented. But, in the matter of the magnitude of the force, the theory demands a force more than $\mathrm{IO}^{30}$ times greater than the force of gravitation really is. This result compels the belief that these Lorentz equations in their present form without modification do not apply to the electrons in the atoms of matter.

However, it is found that, when the expression for the inverse square terms thus derived is multiplied by a factor proportional to the kinetic energy of the electron itself, the correct value of the gravitational pull is obtained. This has given some reason to hope that a modification in the present form of the electromagnetic theory will be found that will make it strictly applicable to the electrons in the atoms when in their steady state not radiating energy. At any rate, I have taken the liberty of introducing the factor demanded by the inverse square terms into the Lorentz forms, thus making a modified form of the present statement of these equations, and find that in so doing the average force between two atoms, at the distances apart concerned in crystals, agrees very closely with the result derived from the older Thomson form of the electromagnetic equations. The first, second, and third terms of the series become ineffective at this range, and, when account is taken of the space-lattice formation of cubic crystals, the series begins with the inverse fourth power, the even powers only following this.

This is precisely the form of equation obtained from the Thomson theory, above referred to, the only difference between the two forms, after introducing the modification, being a factor of 2 in some of the terms in $r^{-4}$. This factor of 2 , however, makes some difference in applying these results to crystals, and, of the two forms, the Thomson equations give consistent results, showing stable equilibrium when we consider the odd planes, as in rock salt, or the even planes as in copper; whereas, the other form gives equilibrium for the odd planes only, but not for the even. The consideration of crystals affords a test between the two forms, and indicates that the Thomson form is to be preferred for these small distances because the results using the odd planes, as in $\mathrm{NaCl}, \mathrm{KCl}$, $\mathrm{KBr}$, etc., fit very closely those using the even planes, as in $\mathrm{Cu}, \mathrm{Fe}, \mathrm{Ag}$, $\mathrm{Pd}$, etc., whereas the other form does not.

The above remarks give some justification for the use of the following equation for the force between two atoms at close range, based upon the Thomson theory. Since the $z$ or $k$ component of the force, that acting 
along the direction of the axis of rotation of the atom, is the only one that we shall require, this is repeated here. The reason that the $i$ and $j$ components are not required is that cubic crystals are so arranged that these components each cancel out in summing the effects of surrounding atoms upon a single selected atom. That is to say, the total force due to all other atoms added together upon the one has the direction of its axis of rotation.

$$
\begin{gathered}
F_{z}=e^{2}\left\{\sum _ { P } ( a \beta ) \sum _ { P ^ { \prime } } ( a \beta ) \left[+\mathrm{I} .5 n \cos \alpha+.75 l \sin \alpha-3.75 m^{2} n \cos \alpha-3.75 l n^{2} \sin \alpha\right.\right. \\
\left.\quad-3.75 l^{2} n \cos \alpha\right] r^{-4} \\
-\sum_{P} a^{2} \sum_{P^{\prime}} a^{2}\left[+5.625 n+3.75 n \sin ^{2} \alpha+7.5 l \sin \alpha \cos \alpha+\mathrm{I} .875^{n} \cos ^{2} \alpha\right. \\
\quad-\mathrm{I} 3 . \mathrm{I} 25\left(+l^{2} n+4 m^{2} n+n^{3} \sin ^{2} \alpha+3 l^{2} n \cos ^{2} \alpha\right. \\
\quad+4 l n^{2} \sin \alpha \cos \alpha+l^{2} n \sin ^{2} \alpha+l^{3} \sin \alpha \cos \alpha+m^{2} n \sin ^{2} \alpha \\
\left.\quad+l m^{2} \sin \alpha \cos \alpha\right) \\
+59.0625\left(+l^{2} n^{3} \sin ^{2} \alpha+l^{2} m^{2} n+l^{4} n \cos ^{2} \alpha+m^{2} n^{3} \sin ^{2} \alpha+m^{4} n\right. \\
\left.\left.\left.\quad+l^{2} m^{2} n \cos ^{2} \alpha+2 l^{3} n^{2} \sin \alpha \cos \alpha+2 l m^{2} n^{2} \sin \alpha \cos \alpha\right)\right] r^{-6}\right\} k .
\end{gathered}
$$

In this equation $e$ is the charge of the electron, $a$ the radius of its orbit, and $\beta$ the ratio of its velocity to that of light. $P$ is the number of electrons in the first, and $P^{\prime}$ in the second atom. The summations indicated in the coefficients are to be extended to each electron in each atom respectively. $l, m$, and $n$ are the direction cosines of the center of the second atom with respect to the center of the first, referred to rectangular axes, $x i, y j$, and $z k$, having the origin at the center of the first atom. The positive direction of the $2 k$ axis is that of the axis of rotation of the first atom, so that the rotation is clockwise viewed from the positive end. The positive direction of the $y j$ axis is then defined by the vector $k \times k^{\prime}$, $k^{\prime}$ being the unit vector in the direction of the axis of rotation of the second atom. This vector takes the direction of the intersection of the equatorial planes of the two atoms, being perpendicular to both $k$ and $k^{\prime}$, and the positive direction is such that rotation from $k$ to $k^{\prime}$ appears counterclockwise viewed from the positive end. The quantities in the brackets are functions of the position of the second atom and the direction of its axis with respect to the first atom. These expressions become numerics as soon as the position of the second atom is known, that is, when the form of the space-lattice of a crystal is specified. The equation may be written $^{1}$

${ }^{1}$ The letter $l$ has inadvertently been employed in two senses here, first as a direction cosine in the functions, $f_{4}$ and $f_{6}$, and then as the cube edge in $l^{-4}$ and $l^{-6}$, but no confusion will arise from this. 
$F_{z}=e^{2}\left\{f_{4}(l, m, n, \alpha) \sum_{P}(a \beta) \sum_{P^{\prime}}(a \beta) l^{-4}+f_{6}(l, m, n, \alpha) \sum_{P} a^{2} \sum_{P^{\prime}} a^{2} l^{-6} \cdots\right\} k$.

The two quantities, $\Sigma_{P}(a \beta)$ and $\Sigma_{P} a^{2}$, are characteristic properties of the atoms, and it is the purpose of this investigation to learn something about them, as far as anything can be ascertained from a study of crystals. In the case of the diamond ${ }^{1}$ an example has been given of the arrangement of the planes and directions of the axes of rotation of each atom, to which reference is made, in which it is found that the equation for the total force on one atom due to the others may be written

$$
F_{z}=e^{2}\left\{-15.7225 \sum_{P}(a \beta) \underset{P^{\prime}}{\Sigma}(a \beta) l^{-4}+252.83 \underset{P}{\sum_{P}} a^{2} \sum_{P^{\prime}} a^{2} l^{-6} \cdots\right\} k,
$$

where $l$ is the edge of the elementary tetrahedron. Since this is $\sqrt{2}$ times the cube edge, the above becomes

$$
F_{z}=e^{2}\left\{-3.9306 \sum_{P}(a \beta) \sum_{P^{\prime}}(a \beta) l^{-4}+3 \mathrm{r} .604 \underset{P}{\sum_{P}} a^{2} \sum_{P^{\prime}} a^{2} l^{-6} \cdots\right\} k,
$$

where $l$ is now the cube edge. When this force is equated to zero for the equilibrium condition, we obtain, putting $d=3 \mathrm{r} .604 / 3.9306=8.04$,

$$
\frac{\sum_{P}(a \beta) \sum_{P^{\prime}}(a \beta)}{\sum_{P} a^{2} \sum_{P^{\prime}} a^{2}}=\frac{d}{l^{2}}=\frac{8.04}{l^{2}} .
$$

A similar process has been carried out for the 124 atoms surrounding the central atom in a simple cubic lattice of edge $4 l$, (see appendix) giving, for the odd planes on one half of the cube only,

$$
F_{z}=e^{2}\left\{0.6013 \sum_{P}(a \beta) \sum_{P^{\prime}}(a \beta) l^{-4}-4.6455 \sum_{P} a^{2} \sum_{P^{\prime}} a^{2} l^{-6} \cdots\right\} k,
$$

and, for the even planes on one side only,

$$
F_{z}=e^{2}\left\{-0.495 \mathrm{I} 7 \sum_{P}(a \beta) \sum_{P^{\prime}}(a \beta) l^{-4}+0.94059 \underset{P}{\sum_{P}} a^{2} \sum_{P^{\prime}} a^{2} l^{-6} \cdots\right\} k .
$$

Equating these to zero gives for the odd planes

$$
\frac{\sum_{P}(a \beta) \sum_{P^{\prime}}(a \beta)}{\sum_{P} a^{2} \sum_{P^{\prime}} a^{2}}=\frac{v}{l^{2}}=\frac{7.73}{l^{2}},
$$

and, for the even planes,

$$
\frac{\sum_{P}(a \beta) \sum_{P^{\prime}}(a \beta)}{\sum_{P} a^{2} \sum_{P^{\prime}} a^{2}}=\frac{u}{l^{2}}=\frac{\mathrm{r} .90}{l^{2}} .
$$

Experimental evidence from measurements with the X-ray spectrometer gives the cube edge, $l$, and the form of lattice, but nothing else in these equations. From such data we obtain merely the ratios in the left

${ }^{1}$ Phil. Mag., Aug., 19r5, p. 257. 
hand members of (5), (8) and (9), the right members becoming simple numbers when the values of $l$ are substituted.

It is at this point where some kind of hypothesis concerning the quantities $a$ and $\beta$, as relating to the atoms, is required. Heretofore, in the papers referred to, the equal moment of momentum hypothesis was introduced for lack of something better, though there seemed to be no very urgent reason why this should be true. The quantity $a \beta$ is proportional to this angular moment of momentum, and, if this has the same value for each electron in the atoms, the quantity in the numerator of the left member of these equations is much simplified, becoming $P P^{\prime}$ times a constant, these being the number of electrons respectively in the atoms. If we should make this assumption, the values of $\Sigma a^{2}$ for the different atoms in the crystals considered might be found in terms, of this constant.

Since, however, we have been able to calculate the speed of rings of electrons, that of four being $\beta=.00846$, and of eight $\beta=.012$, it is evident that the natural tendency of the outer rings is to have a greater actual velocity than the inner rings, if the outer ring has a greater number of electrons. The equal angular moment of momentum hypothesis, however, demands a greater velocity for the smaller ring, the velocity being twice as great at half the radius, and the differential angular velocity between different rings is very pronounced. On the other hand, the calculation of the speed of rings seems to make the angular velocities more nearly equal for different radii. If, for example, the radii were in the ratio of the above speeds, that of the ring of eight being 12 and of the ring of four 8.46 , the angular velocities of the two rings would be the same.

It seems neither safe to assume an equal angular moment of momentum nor an equal angular velocity for each electron in the light of present knowledge. There is another investigation, however, which has a direct bearing upon this matter. The tentative formula for the weight of an atom due to the earth's attraction, to which reference has been made, is

where

$$
F=\frac{\mathrm{I}}{8} \frac{m}{K} m_{0} e_{1} e_{2} \underset{P}{\sum_{P}} \beta^{2} \underset{E}{\Sigma^{2}} \beta^{2} r_{E}^{-2}=k \sum_{\mid P} \beta^{2}
$$

$$
k=\frac{\mathbf{I}}{8} \frac{m}{K} m_{0} e^{2} \gamma_{E}^{-2} \underset{\boldsymbol{E}}{\mathrm{S}} \beta^{2}
$$

the first summation being extended over a single atom, and the second over all the atoms composing the earth, which is, of course, a constant quantity if we are merely comparing the weights of two atoms. $r_{E}$ is the radius of the earth. This result indicates that the weight of an atom is 
strictly proportional to the sum of the squares of the linear velocities of the electrons within the atom. Since the weights of atoms are constant in the same locality, we may better assume that $\Sigma_{P} \beta^{2}$ is constant for a given atom. This quantity, however, does not occur, directly anyway, in the equations (5), (8) and (9), by which we have to investigate crystals.

The solution for the speed of rings of electrons also shows that the linear velocity is independent of the radius of the ring, and, hence, coupling this with the suggestion as to the cause of the weight, we see that it is quite possible that the radii of the orbits of the electrons in an atom may change without affecting the speeds, or consequently, their weights. But, as the radius increases, the angular velocity decreases proportionally in order to maintain the same actual velocity. We may write the three quantities for the atoms, with which we are concerned as follows,

$$
\begin{gathered}
\sum_{P} a^{2}=a_{1}{ }^{2}+a_{2}{ }^{2}+a_{3}{ }^{2}+\cdots a_{P}{ }^{2}, \\
\sum_{P}(a \beta)=\frac{\mathrm{I}}{c}\left(a_{1}{ }^{2} \omega_{1}+a_{2}{ }^{2} \omega_{2}+a_{3}{ }^{2} \omega_{3}+\cdots a_{P}{ }^{2} \omega_{P}\right), \\
\sum_{P} \beta^{2}=\frac{\mathrm{I}}{c^{2}}\left(a_{1}{ }^{2} \omega_{1}{ }^{2}+a_{2}{ }^{2} \omega_{2}{ }^{2}+a_{3}{ }^{2} \omega_{3}{ }^{2}+\cdots a_{P}{ }^{2} \omega_{P}{ }^{2}\right) .
\end{gathered}
$$

For a single ring atom the values of $a$ and $\omega$ are the same for each electron, and, for a ring of $P$ electrons, we have

$$
\begin{gathered}
\sum_{P} a^{2}=P a^{2}, \\
\sum_{P}(a \beta)=\frac{P}{c} a^{2} \omega, \\
\sum_{P} \beta^{2}=\frac{P}{c^{2}} a^{2} \omega^{2} .
\end{gathered}
$$

From these we have, for a single ring atom,

$$
\frac{\Sigma(a \beta)}{\Sigma a^{2}}=\frac{\Sigma \beta^{2}}{\Sigma(a \beta)}=\frac{\omega}{c}, \text { or }[\Sigma(a \beta)]^{2}=\Sigma \beta^{2} \Sigma a^{2} .
$$

For a multiple ring atom we may use a mean square radius, mean velocity, and mean square velocity, giving instead of (I2)-(I4)

$$
\begin{gathered}
{\underset{P}{P} a^{2}}=P a_{0}{ }^{2}, \\
\underset{P}{\Sigma_{P}(a \beta)}=\frac{P}{c} \omega_{1} a_{0}^{2}, \\
\underset{P}{\Sigma_{P} \beta^{2}}=\frac{P}{c^{2}} \omega_{2}^{2} a_{0}^{2} .
\end{gathered}
$$


The subscripts are used in connection with the angular velocities because the two mean velocities obtained in this way are not exactly the same. To show by how much they differ from each other in a special case, an example is given below. We obtain from (19)-(2I)

$$
\begin{gathered}
\frac{\sum_{P}(a \beta)}{\sum_{P} a^{2}}=\frac{\omega_{1}}{c} \\
\frac{\sum_{P} \beta^{2}}{\sum_{P}(a \beta)}=\frac{\omega_{2}^{2}}{c \omega_{1}} .
\end{gathered}
$$

In a two-ring atom, let the radius of the outside ring be $a$, speed such that $\beta=.012$, and number of electrons eight. For the inside ring let $a_{i}=.402 a, \beta_{i}=.00846$, and the number of electrons four. Then, for the atom

$$
\begin{aligned}
& \sum_{P} a^{2}=8 a^{2}+4(.402 a)^{2}=8.6464 a^{2}=P a_{0}^{2}=\mathrm{I} 2 a_{0}^{2}, \\
& \sum_{P}(a \beta)=8 a \times .012+4 \times .402 a \times .00846=.1096 a \\
& =\frac{P}{c} \omega_{1} a_{0}^{2}=\frac{\mathrm{I} 2}{c} \omega_{1} a_{0}^{2}, \\
& \sum_{P} \beta^{2}=8 \times .012^{2}+4 \times .00846^{2}=.001438=\frac{P}{c^{2}} \omega_{2}^{2} a_{0}{ }^{2}=\frac{\mathrm{I} 2}{c^{2}} \omega_{2}^{2} a_{0}^{2}, \\
& \frac{\sum_{P}(a \beta)}{\sum_{P} a^{2}}=\frac{.01268}{a}=\frac{\omega_{1},}{c} \\
& \frac{\sum_{P} \beta^{2}}{\sum_{P}(a \beta)}=\frac{.01312}{a}=\frac{\omega_{2}^{2}}{c \omega_{1}}
\end{aligned}
$$

whence

$$
\left(\frac{\omega_{2}}{\omega_{1}}\right)^{2}=\frac{.01312}{.01268}=1.034 \text {, and } \frac{\omega_{2}}{\omega_{1}}=1.017 \text {. }
$$

According to this example we do not make much error in assuming that equation (I8), which is strictly true for the single ring atom, is also approximately true for more complicated forms. Making this approximation, (5) becomes

$$
\sum_{P} a_{P^{\prime}}^{2} a^{2}=\frac{l^{4}}{d^{2}} \underset{P}{\Sigma_{P}} \beta_{P^{\prime}}^{2 \beta^{\prime}} \beta^{2}
$$

with similar expressions for (8) and (9), the differences being in the values of the constants.

But, by (Io), we may consider that $\Sigma_{P} \beta^{2}$ is constant for a given atom, and thus may find its value from the atomic weight. If $W$ is the atomic 
weight in dynes, and $A$ the atomic weight referred to oxygen as I6, then $W$ is equal to $A$ times the weight of the hydrogen atom divided by 1.008. Taking the mass of the hydrogen atom as $1.64 \times 10^{-24}$ grams, and its weight $1.64 \times 981 \times 10^{-24}$ dynes, the weight of any atom is

Hence

$$
W=\mathrm{I} 5.96 \times 1 \mathrm{IO}^{-22} A_{P}=k \mathrm{\Sigma}_{P} \beta^{2}, \text { by (Io). }
$$

where

$$
\sum_{P} \beta^{2}=\mathrm{I} 5.96 \times \mathrm{IO}^{-22} A_{P} / k=k_{1} A_{P},
$$

$$
k_{1}=\mathrm{I} 5.96 \times \mathrm{Io}^{-22} / k .
$$

Putting this in (30) gives as the equilibrium condition for the diamond form of lattice,

$$
\underset{P}{\sum_{P}} a^{2} \sum_{P} a^{2}=\frac{k_{1}^{2}}{d^{2}} A_{P} A_{P} l^{4}
$$

For a crystal like rock salt we obtain from (8) a similar form to this, with the constant ${ }^{1} d$ equal to 7.73 instead of 8.04 , being obtained from the odd planes of the simple cubic lattice, and for copper from (9) a similar form with the constant I.90, obtained from the even planes of the simple cubic lattice, in this case the even planes being the only ones present.

These formulæ have been applied to twenty different crystals belonging to the cubic or isometric system, some of which are known to have been examined by the spectrometer and others not. In certain cases the formulæ give $\Sigma a^{2}$ directly, when there is but one kind of atom in the crystal, as diamond, copper, silver, iron, etc., but in most cases they give products $\Sigma_{P} a^{2} \Sigma_{P^{\prime}} a^{2}$ for the two kinds of atoms that enter the crystal. The separate values in this case for each kind of atom may only be obtained when one of them is known from some other crystal.

The remarkable result is obtained that $\Sigma a^{2}$ shows a gradual progression from element to element, the irregularities being of about the same order as the irregularities in atomic weights. The curves obtained from these twenty crystals, containing as many separate kinds of atoms altogether, are shown in Figs. I and 2. The best values of $\Sigma a^{2}$ and $\Sigma(a \beta)$ to agree with the different crystals are plotted for each of the twenty elements as separate points.

It is found, however, that, in order to make the points fall near the curves, the calculation must be made for some of the crystals as though there were double instead of single atoms at each point of the space lattice. The points either fall near the curve for the correct form of lattice and proper number of atoms, or very far from it for a wrong

1 See equation (5) above. 
combination. The compounds of sulphur may serve as an illustration. The crystals, zincblende ( $\mathrm{ZnS}$, diamond lattice) and galena ( $\mathrm{PbS}$, rock salt lattice), have been studied by the spectrometer, and the measured spacings of the planes agree with those calculated from their densities, on the assumption that there is but a single atom of zinc and a single atom of sulphur at the points of the lattice. The points, as calculated by the above formulæ with this spacing, do not fall anywhere near the curve, but, if we had calculated the lattice on the assumption that the atoms of each were double at the various points, we should have obtained an edge for the elementary cube $2^{1 / 3}$ greater than before, and the product $\Sigma_{P} a^{2} \Sigma_{P^{\prime}} a^{2}$ would have been $2^{4 / 3}$ greater, depending upon the fourth power of $l$. The points for zinc and sulphur thus obtained fall near these curves.

TABLE I.

List of Crystals and Elements from Which the Curve in Fig. $\mathrm{I}$ is Obtained.

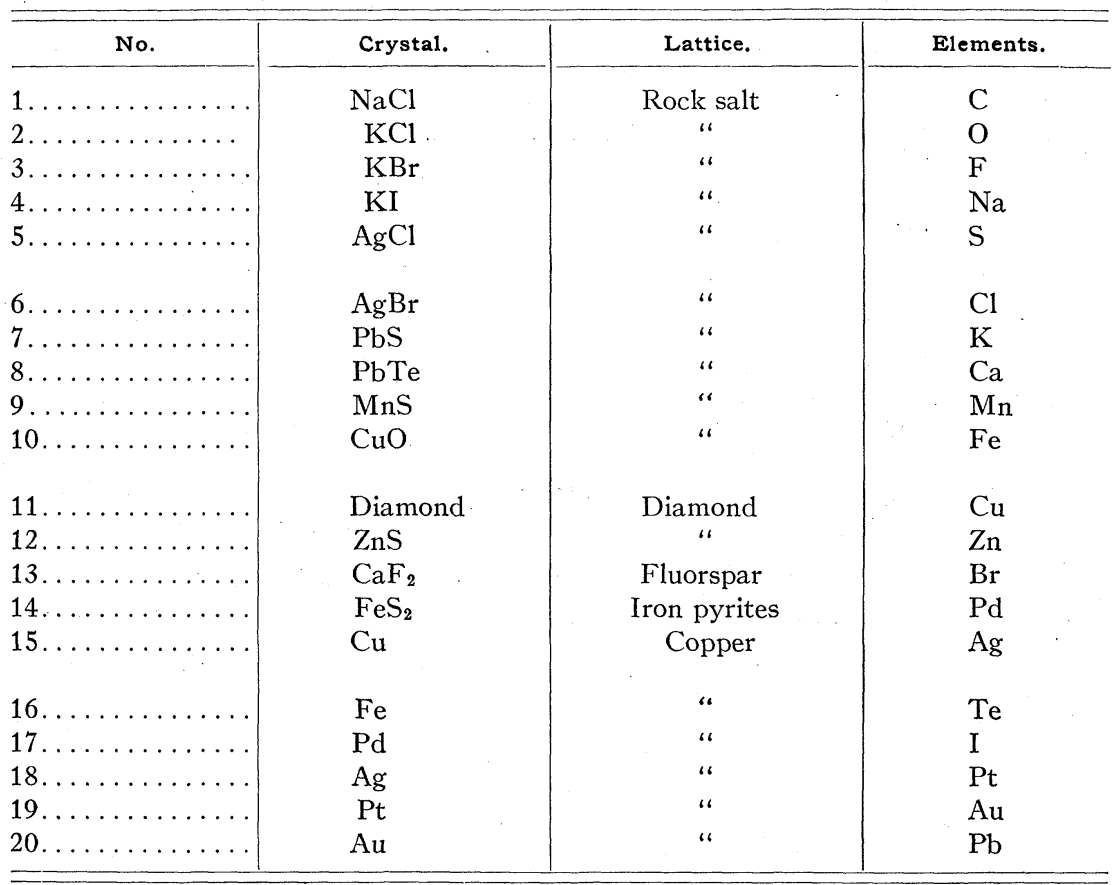

In the case of galena we have to assume four atoms of lead and four of sulphur instead of one at the points of the lattice, making $l$ greater by $2^{2 / 3}$, and $l^{4}$ by $2^{8 / 3}$, than it really is to make the points fall near the curves. Since the spectrometer has definitely settled the question of the length of the elementary cube of the space lattice, we can not suppose that 
there are double atoms in zincblende and quadruple atoms in galena at the points of the lattice, and, according to the hypothesis that $\Sigma \beta^{2}$ is proportional to the weight, we are led to believe that $\Sigma a^{2}$ is not always the same in some kinds of atoms at least.

If we consider that manganblende, $\mathrm{MnS}$, is a simple cubic lattice like rock salt, the values of $\Sigma a^{2}$ fall near the curve by assuming double atoms.

Another crystal containing sulphur is iron pyrites, which has been described by W. H. and W. L. Bragg in "X-rays and Crystal Structure " in much detail. If we apply the same equilibrium formula to this as applies to diamond, zincblende and fluorspar, we obtain points close to the curve by assuming double atoms. How it is possible that the same equilibrium condition may be applied to such different lattices is shown in the appendix hereto.

The point we are now making is that these four crystals fall into line if we admit that there are two different kinds of sulphur atoms, the same kind of sulphur in $\mathrm{ZnS}, \mathrm{MnS}$, and $\mathrm{FeS}_{2}$, and a different kind in $\mathrm{PbS}$, their weights being the same in each case, but $\Sigma a^{2}, \Sigma(a \beta)$ and $\omega_{1}$ differing for the two kinds. To fall upon the curve in Fig. I, the sulphur atom should have approximately

$$
\underset{P}{\Sigma_{P}} a^{2}=300 \times 10^{-16} \frac{k_{1}}{v} .
$$

In $\mathrm{ZnS}, \mathrm{MnS}$, and $\mathrm{FeS}_{2}$ the value required is $\left(\frac{1}{2}\right)^{4 / 3}=.397$ of this, and in $\mathrm{PbS}\left(\frac{1}{2}\right)^{8 / 3}=. \mathrm{I} 575$ of it. The value corresponding to the curve does not occur in these crystals, but it seems likely that it does occur sometimes, perhaps in other crystals.

A similar statement may be made for the atoms of oxygen, chlorine, and bromine. By admitting two possible forms for these three atoms, having the same atomic weight in each case, all of the twenty crystals considered are brought into line. Chlorine and bromine in the halogens, $\mathrm{NaCl}, \mathrm{KCl}, \mathrm{KBr}$ have values of $\Sigma a^{2}$ corresponding to the curve, but, in $\mathrm{AgCl}$ and $\mathrm{AgBr}$, the $\mathrm{Cl}$ and $\mathrm{Br}$ have values $\left(\frac{1}{2}\right)^{4 / 3}=.397$ of that in $\mathrm{KCl}$ and $\mathrm{KBr}$. In the mineral melaconite, $\mathrm{CuO}$, the oxygen has a value of $\Sigma a^{2}\left(\frac{1}{2}\right)^{4 / 3}$ of that given by the curve. This is the only crystal considered containing oxygen. It seems likely that a value of oxygen will be obtained from other crystals which agrees with the curve. The only crystal containing fluorine, $\mathrm{CaF}_{2}$, gives a value close to the curve. We seem justified in expecting to find in other crystals a value equal to $\left(\frac{1}{2}\right)^{4 / 3}$ of this for $F$.

In Fig. 2 the atomic weights of the elements are plotted with reference to the atomic numbers, and a mean curve drawn through the points. By equation (32) we may obtain from this the values of $\Sigma \beta^{2}$ when the 
abscissæ of this curve are multiplied by the constant $k_{1}$. The approximate value of $\Sigma(a \beta)$ may then be derived by multiplying together the corresponding abscissæ of $\Sigma a^{2}$ in Fig. $\mathrm{I}$ and $\Sigma \beta^{2}$, and taking the square root of the product, according to (I 8$)$. The curve of $\Sigma(a \beta)$, thus obtained, is shown in II., Fig. 2. And, according to (22), we may obtain the mean angular velocity of revolution of all the electrons in the atoms by dividing $\Sigma(a \beta)$ by $\Sigma a^{2}$, which is shown in Curve III., Fig. 2.

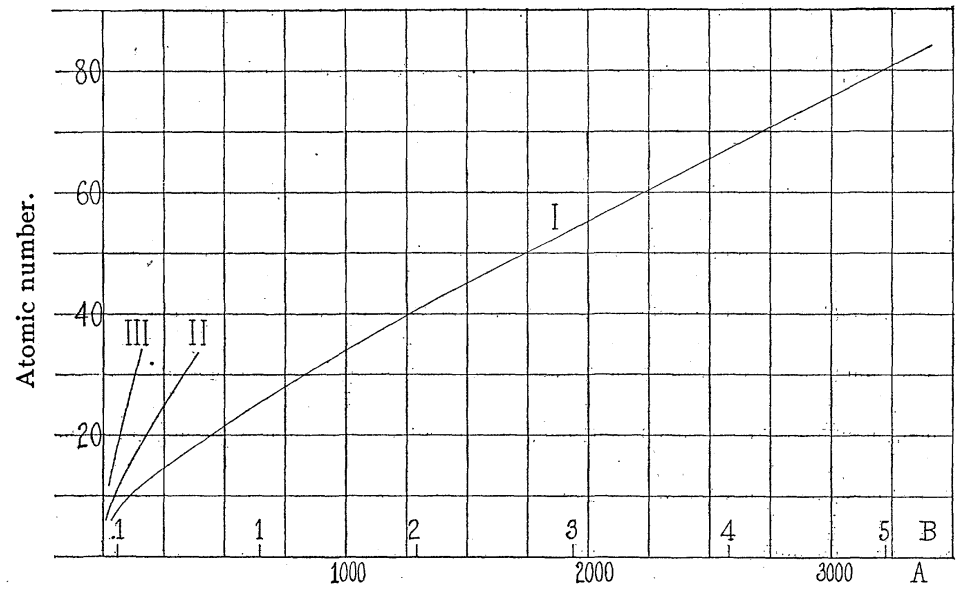

Fig. 1.

Curves I., II. and III. $\frac{v}{k_{1}} \underset{P}{\sum} a^{2} \times 10^{16}$. Scale $A$.

Curves I., II. and III. $\underset{P}{\sum} a^{2} \times 10^{18}$ sq. $\mathrm{cm} . \quad$ Absolute scale $B$.

The character of the curve of $\Sigma a^{2}$, Fig. I, supports the theory that the electrons are distributed in rings resembling those originally calculated by Thomson, and that the volume of the sphere enclosing the rings increases by uniform steps as electrons are added. The volume of a sphere enclosing the orbits of the electrons, on the assumption that they are arranged in a plane, may be taken roughly proportional to the cube of the mean radius. The mean square radius is proportional to $\Sigma a^{2} / N$, where $N$ is the atomic number, and the volume, therefore, proportional to $\left(\Sigma a^{2} / N\right)^{3 / 2}$. If the volume is also proportional to the number of electrons, and this again to the atomic number, we derive the equation

Hence

$$
\left(\frac{\Sigma a^{2}}{N}\right)^{3 / 2}=N \text { times a constant }
$$

$$
\Sigma a^{2}=b N^{5 / 3} \text {, where } b \text { is a constant. }
$$

The values of $\Sigma a^{2}$ read from the curve, and $N^{5 / 3}$ calculated from the corresponding atomic numbers, are as follows: 
TABLE II.

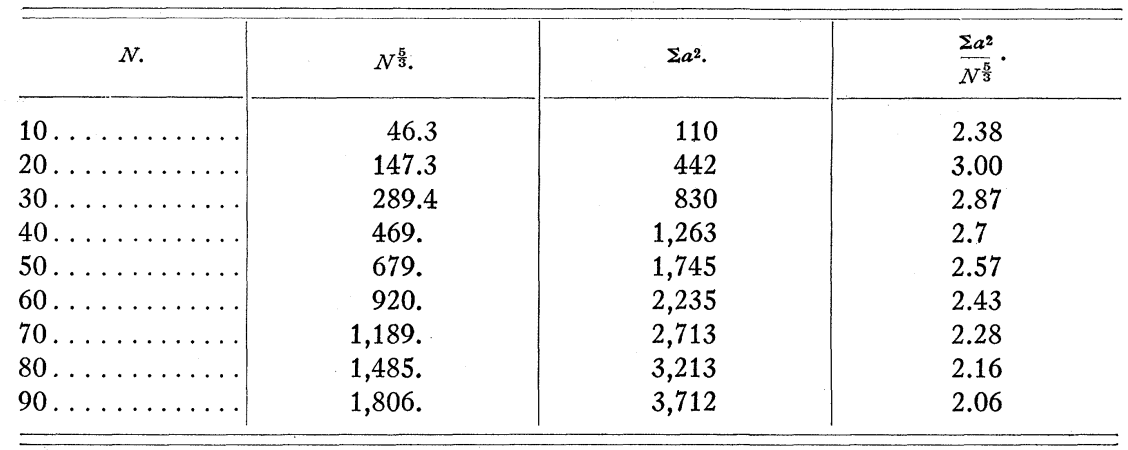

The constancy of this ratio in the above table is as nearly perfect as we should expect, were it exactly true that the volume of a sphere enclosing the outside rings increases by equal steps for the addition of each electron, because of the approximation we are forced to use in deriving the result in (35).

The curve for $\Sigma(a \beta)$ in Fig. 2 is very nearly a straight line for the atomic numbers above 40, Zirconium; but the line does not pass through the origin. If we dvide $\Sigma(a \beta)$ by $N$, to obtain the average moment of momentum per electron, these exhibit a gradual increase with increasing atomic numbers. The average for the heavier elements is sufficiently constant to have suggested the idea that the angular moment of momentum for each electron is constant, ${ }^{1}$ but, in the lighter elements, these values vary considerably from those in the heavy elements.

The curve for the mean angular velocity of each atom, III., Fig. 2, shows a decrease in the frequency for an increase in the atomic number. The frequency becomes very large for the lightest elements. For carbon this frequency, as we shall see by the two examples, is about $.78 \times 10^{18}$. The average frequencies for the other elements are obtained from the ratio of the abscissæ of Curve I. to those of Curve II., Fig. 2, and, since Curve II. approaches zero much more rapidly than Curve I., the ratio and the frequency become rapidly larger for decreasing atomic numbers. The fundamental value for hydrogen has been found elsewhere ${ }^{2}$ to be of the order of $\mathrm{IO}^{20}$, considerably greater than that for carbon.

If one identifies the frequencies of revolution of the electrons in their orbits with optical frequencies according to Bohr's theory, the radii of the orbits, being inversely as the frequencies, come out much greater than the orbits indicated by the above average values of the frequencies.

1 Moseley, Nature, Jan. I5, I9I4, and F. A. Lindemann, Nature, Jan. I, Feb. 5, I9I4.

2 Unpublished. 
It is possible, of course, that a small number of electrons circulate in large orbits with smaller frequencies, and that the rest have very much smaller orbits and higher frequencies, so far as we can tell from these average values. If this is true, then the electromagnetic forces, as developed in infinite series cannot be applied to these outside electrons, because the force-series becomes non-convergent when the distance between the centers is comparable with the diameter.

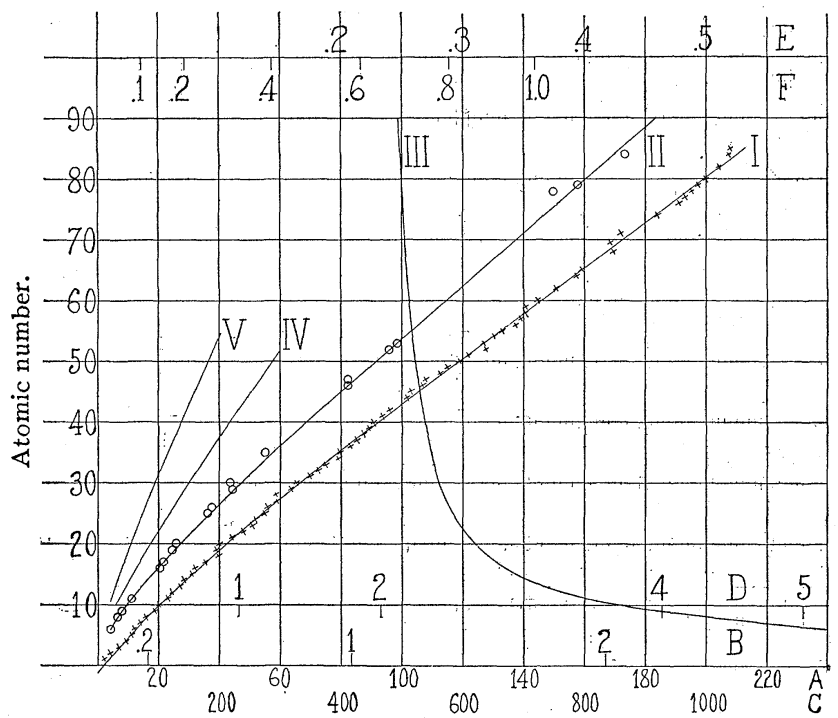

Fig. 2.

Curve I. $\frac{\mathrm{I}}{k_{1}} \sum_{P} \beta^{2}$. Scale $A . \quad$ Curve I. $\underset{P}{\sum} \beta^{2} \times \mathrm{I} \mathrm{O}^{2}$. Absolute scale $B$.

Curves II., IV. and V. $\frac{\sqrt{v}}{k_{1}} \sum_{P}(a \beta) \times 10^{8} . \quad$ Scale $C$.

Curves II., IV. and V. $\underset{P}{\sum}(a \beta) \times$ I0 $0^{10}$. Absolute scale $D$.

Curve III. $\frac{\mathrm{I}}{\sqrt{v}}\left(\frac{\Sigma \beta^{2}}{\Sigma(a \beta)}\right)=\left(\frac{\Sigma(a \beta)}{\Sigma a^{2}}=\frac{\omega_{1}}{c}\right) \times$ I0 $^{-8}$. Scale $E$.

Curve III. $\frac{\omega_{1}}{c} \times \mathrm{ro}^{-8} . \quad$ Absolute scale $F$.

There have been cogent reasons for believing that a few so-called "valency" electrons do have these larger orbits, and, if so, electromagnetic theory should still be capable of dealing with the matter as long as they are in the steady state not radiating energy. A more comprehensive method of analysis is required, however, before any equations embracing these outside electrons can be obtained. It seems to be necessary to show that such electrons may be permitted by the theory 
without disturbing the equilibrium, on account of their mutual interference and the resulting perturbations produced. The evidence in favor of their existence is greatly strengthened by the very recent work of J. Frenkel ${ }^{1}$ in calculating on Bohr's theory the "intrinsic potentials" of, and the Volta contact electromotive forces between, metals and nonmetals by means of the supposed existence of a small number of electrons having these large orbits. In the way it is done, it is these large orbits alone that are responsible for the effects, the small ones being of no avail. He also calculates the electrical energy of the quasi-surface condenser thus produced, making it the same as the well-known energy of surface tension, which is thus explained in terms of atomic structure.

It is noteworthy that the order of magnitude of the frequencies in Curve III., $\mathrm{IO}^{18}$, is the same as that of characteristic X-ray frequencies. It has been pointed out before that these frequencies of revolution should not be related directly to these $\mathrm{X}$-ray frequencies. The one may be a function of the other, but the evidence goes to show that the $\mathrm{X}$-ray frequencies are functions primarily of the atomic number and a series of ordinals alone, which would give the smooth character to the Moseley curves. The dependence upon $\Sigma a^{2}, \Sigma(a \beta)$ and rotation frequency secondarily may account for the small curvature observed in his curves. The large number of lines in the $\mathrm{X}$-ray spectrum is alone almost sufficient to make this independence of the two kinds of frequencies probable.

\section{The Absolute Values of the Constants. ${ }^{2}$}

The curves in Figs. I and 2 have abscissæ which are dependent upon the absolute value of the constant $k_{1}$, defined by the equations (33) and (II) above. It is necessary to know the value of this constant before $\Sigma a^{2}$, etc., can be found in absolute measure. It is possible to find $k_{1}$ if we know the number of electrons in any one atom, their arrangement in rings, and their speeds. We shall make a tentative assumption as to the carbon atom, and derive from it the value of $k_{1}$. The reason for giving the curves in terms of this constant is so that, if any one prefers a different assumption as to carbon or any other atom, the absolute values may be more readily obtained. Let us take $\Sigma \beta^{2}$ for carbon as given in the example, equation (26). Then, by (32)

${ }^{1}$ J. Frenkel, On the Surface Electric Double-layer of Solid and Liquid Bodies, Phil. Mag., April, 19I7, p. 297.

2 The following numerical estimates of absolute values must, of course, be considered as tentative and subject to revision. They are chiefly based upon the calculation of actual velocities of electrons in rings according to electromagnetic theory. The process of making such calculations is long and tedious and should be checked both as to method and errors of a mechanical nature. It should be emphasized that the methods outlined here should yield the proper numerical values when these velocities of electrons in rings are accurately known. 


$$
k_{1}=.001438 / 12=1.198 \times 10^{-4} .
$$

The value of $v$, as in (8), is 7.73. Hence, to convert the abscissæ of the curves into absolute measure, multiply those of

Curve I., Fig. I, for $\Sigma a^{2}$ by $k_{1} \times 10^{-16} / v=0.155 \times 10^{-20}$,

Curve I., Fig. 2 , for $\Sigma \beta^{2}$ by $k_{1}=$ I.198 $\times 10^{-4}$,

Curve II., Fig. 2, for $\Sigma(a \beta)$ by $\frac{k_{1}}{\sqrt{v}} \mathrm{IO}^{-8}=0.43 \mathrm{I} \times \mathrm{IO}^{-12}$,

Curve III., Fig. 2, for $\frac{\Sigma \beta^{2}}{\Sigma(a \beta)}=\frac{\Sigma(a \beta)}{\Sigma a^{2}}=\frac{\omega_{1}}{c}$ by $\sqrt{v} \times 10^{8}=2.78 \times 10^{8}$.

For the carbon atom the reading from the curve for $\Sigma a^{2}$ is 34.95 . Multiplying by the factor $0.155 \times 10^{-20}$ gives in absolute measure $\Sigma a^{2}=5.42 \times 10^{-20}$ sq. $\mathrm{cm}$. Equating this to (24) gives the radius of the outside ring $a=0.792 \times 10^{-10} \mathrm{~cm}$. This absolute value for the radius is in accord with former results, all of which show that the radius is a very small quantity compared with the distance between adjacent atoms. The edge of the tetrahedron in diamond is $2.528 \times 10^{-8} \mathrm{~cm}$., 3 I9 times the radius above determined.

We obtain also the mean angular velocity and frequency of revolution for carbon from $(25)$ to be $\omega=4.80 \times \mathrm{IO}^{18}$, and $n=0.764 \times \mathrm{IO}^{18}$.

Had we made a different assumption for the carbon atom, the order of magnitude of these quantities is not greatly changed. For example, let the carbon atom be supposed to consist of a single ring of six electrons, for which $\beta$ falls between .00846 and .or2, say at .oro. The exact value of $\beta$ for a ring of six has not been calculated at this writing. From this assumption $\Sigma \beta^{2}=6 \times 10^{-4}$, and

$$
k_{1}=0.5 \times \mathrm{IO}^{-4} .
$$

Hence $\left(k_{1} \times \mathrm{IO}^{-16}\right) / v=6.47 \times \mathrm{IO}^{-22}$, and $\Sigma a^{2}=6.47 \times 34.95 \times \mathrm{IO}^{-22}$ $=2.26 \times \mathrm{IO}^{-20} \mathrm{sq} . \mathrm{cm}$., and the radius of the ring $a=.6 \mathrm{I} 4 \times \mathrm{IO}^{-10}$ $\mathrm{cm}$., instead of .792 according to the former assumption. Also $\Sigma(a \beta)=6 a \beta=3.684 \times 10^{-12}$, and

$$
\frac{\Sigma \beta^{2}}{\Sigma(a \beta)}=\frac{\Sigma(a \beta)}{\Sigma a^{2}}=\frac{6 \times 10^{-4}}{3.684 \times 10^{-12}}=\frac{3.684 \times 10^{-12}}{2.26 \times 10^{-20}}=1.63 \times 10^{8}=\frac{\omega}{c} .
$$

Hence the angular velocity $\omega=4.89 \times 10^{18}$, about the same as the mean value of $\omega$ in the former example.

Speculation as to the Kind of Atoms in the Interior of the Earth.

By means of the absolute values of $k_{1}$ in (36) and (37), obtained from different assumptions as to the structure of the carbon atom, we are enabled to get an approximate value of the constant $k$ in the weight 
equation (Io). For, by (33) $k=\mathrm{I} 5.96 \times 10^{-22} / k_{1}$. For the first kind of carbon atom we have

and for the second

$$
k=\mathrm{I} 3.32 \times \mathrm{IO}^{-18},
$$

$$
k=3 \mathrm{I} .9 \times \mathrm{IO}^{-18} \text {. }
$$

A knowledge of this constant enables us to find from (II) $\Sigma_{E} \beta^{2}$ for the earth, that is, the sum of the squares of $\beta$ for every electron in the earth. This is

$$
\underset{E}{\Sigma} \beta^{2}=\frac{8 k r_{E}^{2}}{m_{0} e^{2}},
$$

where $r_{E}$, the radius of the earth may be taken as $6.367 \times 10^{8} \mathrm{~cm}$., and $m_{0}$, the mass of the electron, as $.898 \times 10^{-27} \mathrm{grams}$, and $e=4.77 \times 10^{-10}$ electrostatic units. With the first kind of carbon atom we get

and with the second

$$
\sum_{E} \beta^{2}=21.12 \times 10^{46}
$$

$$
\sum_{E} \beta^{2}=50.56 \times 10^{46} .
$$

Dividing this by the total number of electrons in the earth, we obtain a value of $\beta$ for the average electron in the earth. The volume of the earth is $1.083 \times 10^{27}$ c.c., mean density $5.5247 \pm .0013$, and mass, therefore, $5.984 \times \mathrm{IO}^{27}$ grams.

If we take the number of electrons per atom as proportional to the atomic number, then the number of electrons per gram of any substance is constant. This may be shown as follows. It is well known that a cubic $\mathrm{cm}$. of a perfect gas, under the standard conditions of temperature and pressure, contains the same number of molecules, say $N$. If $d$ is the density of the gas, then the number of molecules per gram is $N / d$, since $\mathbf{I} / d$ is the volume of a gram of the gas. If $M$ is the molecular weight,

$$
M=n_{1} A_{1}+n_{2} A_{2}+n_{3} A_{3}+\cdots,
$$

where $A_{1}, A_{2}$, etc., are the atomic weights of the various atoms in the molecule, and $n_{1}, n_{2}$, etc., the numbers of these atoms respectively. If the number of electrons in the atom, $P$, is proportional to the atomic number or approximately to the atomic weight, we have

$$
A_{1}=b P_{1} ; \quad A_{2}=b P_{2} \text {, etc. }
$$

Hence $M=b\left(n_{1} P_{1}+n_{2} P_{2}+\cdots\right)=b P$, where $P$ is now the number of electrons in one molecule. It follows that the mass of all the molecules in one c.c. of the gas is $m=$ vol. $\times$ density $=d=h N M=k N P$, where $h$ and $k$ are constants. Hence, for two different gases

$$
d / d^{\prime}=P / P^{\prime} \text {. }
$$


Since the number of molecules per gram is $N / d$, the electrons per gram are proportional to $N M / d$ and to $N P / d$. And, since $N$ is constant, and $P / d=P^{\prime} / d^{\prime}$, the electrons per gram are the same for different gases.

The number of electrons per gram is, therefore, the same for all forms of matter, liquids and solids, because the atoms have the same weight in any of these forms, and the number of electrons per atom may be supposed to be the same under all conditions.

Knowing that the number of atoms of hydrogen per gram is $6.05 \times 1 \mathrm{I}^{23}$ approximately, and considering that the hydrogen atom has but a single electron, this number may be taken as the number of electrons per gram for any substance.

Multiplying the electrons per gram by the mass of the earth in grams gives the total number of electrons in the earth as approximately

$$
6.05 \times 1 \mathrm{IO}^{23} \times 5.984 \times 1 \mathrm{IO}^{27}=36.2 \times 1 \mathrm{IO}^{50} .
$$

The mean values of $\beta^{2}$ and $\beta$ for the average electron in the earth may now be found by dividing $\Sigma_{E} \beta^{2}$ by the total number of electrons, giving, for the twelve-electron carbon atom,

$$
\beta^{2}=0.5856 \times 10^{-4} \text { and } \beta=0.00765 .
$$

Comparing this value with $\beta$ for a ring of four electrons, 0.00846 , and for a ring of eight, 0.012 , shows that it is a little less than the value for the ring of four. Comparing with the values of obtained for hydrogen in its different conditions, namely, $0.00738,0.00369$ and 0.00246 , corresponding to the first, second and third states of hydrogen respectively, shows that it is about the same as the value in the first state of hydrogen. The majority of the electrons in the atoms of the earth's crust with which we are acquainted have, according to the Curve I., Fig. 2, a value of $\beta$ considerably in excess of 0.00765 , so that the electrons at the center of the earth must have a value less than the mean. Such a value would be too small to agree with any of the forms of atom except hydrogen. It has been pointed out elsewhere that there is no evidence for the existence of hydrogen in the first state, and that the normal condition for hydrogen is the second state in which $\beta=0.00369$, which is less than the mean value for the earth. If the interior of the earth were composed of hydrogen, the density might still be very large, as this is due to the compactness, or the interspace between atoms, rather than to the character of the atoms themselves. We know that the density at the center must be in excess of 5.52, the mean value, because the average density of the surface, that of the earth's crust, is considerably less than the mean density. 
The above is at least an interesting speculation indicating that we have obtained a method that may eventually yield some information as to the nature of the large body of atoms in the interior of the earth. Had we carried through the calculation based upon the six-electron atom the mean value of $\beta$ would have been about half again larger than that given.

\section{Bulk Modul.I.}

In a former paper ${ }^{1}$ a formula was given by which the bulk modulus of a crystal can be calculated from a knowledge of the forces interacting between the atoms. This may be defined as the ratio of the pressure per sq. $\mathrm{cm}$. in grams weight to the change in volume per unit volume, that is, the substance is more incompressible the greater its bulk modulus. This formula is

$$
M=\frac{\sqrt{3}}{9 \times 98 \mathrm{r}} \frac{\mathrm{r}}{l^{2}} \frac{d F}{d l} .
$$

Table III. gives the values of $M$ for several crystals as calculated by this formula. The measured values of those that are known are given in the third column, together with some crystals for which the modulus has not been calculated, in order to show the great range of measured values for different crystals, as well as to show that the order of magnitude of the range of calculated values is the same.

The calculated values are larger in every instance where the value has been measured, indicating a more incompressible substance than it is measured to be. The range of values among those calculated is about six to one from greatest to least, while the range of the measured values is 66 to one. The measured value for tourmaline is well toward the top of the list of calculated substances, showing the same order of magnitude.

Measurements of the bulk modulus must be subject to considerable error because we have no means of compressing a substance except to press upon it with another substance having a similar character. In such a case it is difficult to say that the two substances do not interpenetrate each other to a certain extent at the surface at least. By exerting pressure by mercury upon a piece of steel, for example, Bridgeman has shown that it is possible to force the mercury completely through the steel, so that, when broken afterwards, it shows an amalgamated surface over the entire break. If any interpenetration whatever occurs it will have the effect of reducing the value of the bulk modulus obtained, which is in line with the results shown in the table. Moreover, if the same substance is used to produce the compression for a number of different substances, the amount of such interpenetration should vary widely with the kind

${ }^{1}$ Loc. cit. 
of space lattice of the crystal. Hence, great variations in measurements should be anticipated if there occurs any interpenetration, which is again in agreement with the results in the table.

TABLE III.

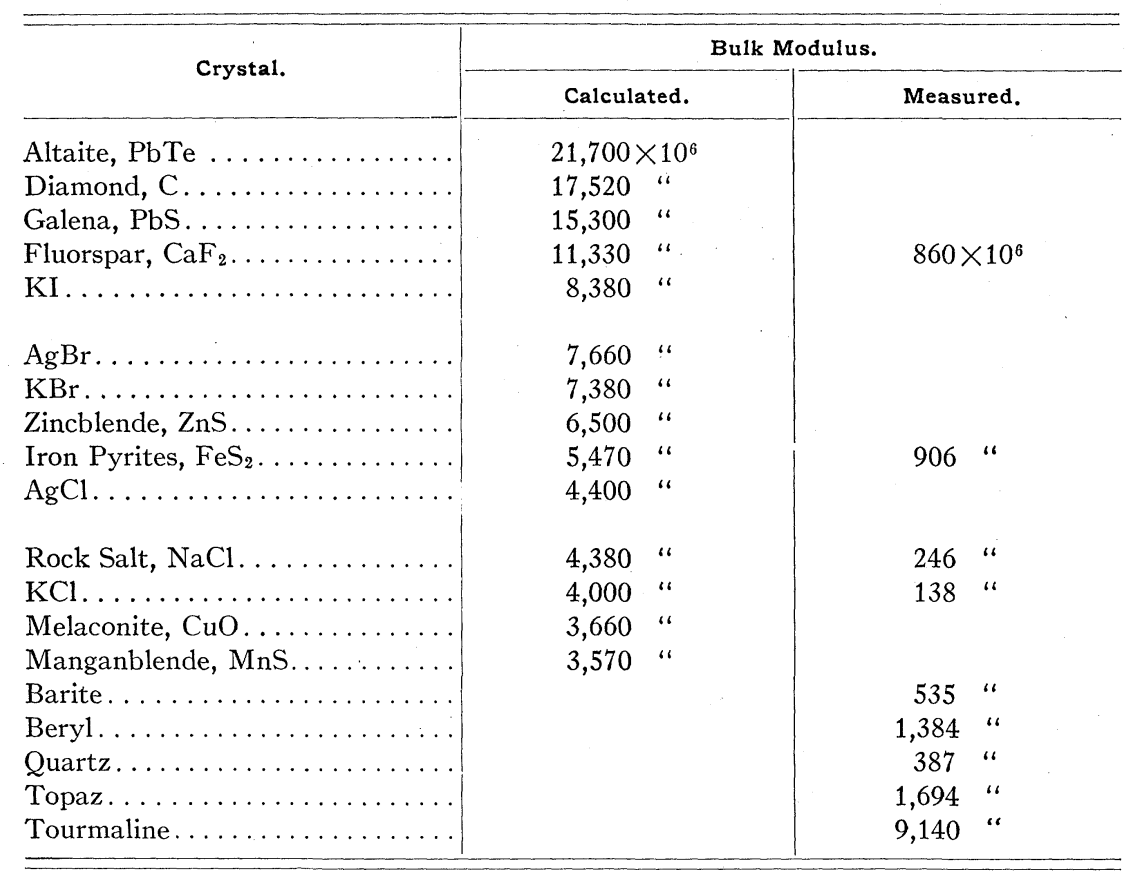

If the same crystal were measured with compressing substances which vary as widely as possible in their properties, it might prove to be the case that different values of the bulk modulus would be obtained for the same crystal, which would help to confirm the above suggestions.

\section{Review AND Summary.}

I. The problem of finding the average mechanical force that one electrical charge exerts upon a second charge, each being in uniform circular motion, has been solved, both for the form of electromagnetic equations originally proposed by Thomson, and for the more recent form of Lorentz involving retarded potentials. It was shown in a former paper that the Lorentz form without modification cannot be applicable to the electrons in the atoms of matter, because their application would produce a force, varying inversely as the square of the distance between two pieces of matter at a great distance apart, more than $10^{30}$ times greater than the existing force of gravitation. When, however, the result thus obtained 
is multiplied by a factor proportional to the kinetic energy of the electron itself, the attraction agrees very closely with that of gravitation. When such a factor is introduced into the Lorentz equations the average force, at the distances considered in crystals, reduces to very nearly the same form whether the Lorentz or the original Thomson equations are used, the same within a factor of 2 in some of the terms in $r^{-4}$. This is regarded as some justification for using the original Thomson equations for these ranges of distance. The equation for the force between two atoms, derived from these equations as given in a former paper, is here applied to twenty crystals belonging to the cubic system.

2. Equilibrium conditions are derived for several forms of space lattice. In each of them the only unknown quantities are $\Sigma_{P} a^{2}$ and $\Sigma_{P}(a \beta)$, summed for each electron in the atom concerned, $a$ being the radius of its orbit, and $\beta$ its speed in terms of the velocity of light.

3. Some hypothesis is required before either the relative or the absolute values of $a$ and $\beta$, the unknown quantities pertaining to the atoms, can be found. In a former paper the equal moment of momentum hypothesis for each and every electron in the atoms was adopted for the lack of something better. This hypothesis led to certain serious difficulties, in that it demanded that in such crystals there should be double atoms instead of single atoms at each point of the space lattice. The evidence of the spectrometer has made this view untenable.

4. The hypothesis as to the atoms adopted in this paper, instead of the equal moment of momentum hypothesis, is that $\Sigma_{P} \beta^{2}$ is constant for any given atom. A former work has indicated that the gravitational attraction between bodies at a distance is proportional to the product of the sum of the squares of the speeds of the electrons summed over each body separately. Since the weight of an atom is constant, it is reasonable to suppose in view of the above that the sum of the squares of the speeds of the electrons within it is constant.

5. This hypothesis avoids the difficulties in which the equal moment of momentum hypothesis involved us, by which $\Sigma_{P}(a \beta)$ is constant for a given atom. According to the new " $\Sigma_{P} \beta^{2}=$ a constant" hypothesis, the same atom may take two or three different forms without altering $\Sigma_{P} \beta^{2}$. This means that the moment of momentum is not constant, but may have two or three different forms corresponding to changes in the radii.

6 . The values of $\Sigma_{P} a^{2}, \Sigma_{P}(a \beta)$ and $\Sigma_{P} \beta^{2}$ have been found for each of twenty different atoms that enter as many different crystals, and are plotted as curves in Figs. I and 2, against the atomic number in terms of a constant multiplier. If an assumption is made as to some one form of atom, these constants may be determined and the above values found for 
any atom in absolute measure. The assumption that must be made concerns the number of electrons in some atom and the speed of each; but, fortunately, the radii of their orbits is not required. It is also fortunate that the speed of electrons in rings has been previously determined numerically for a ring of 4 and a ring of 8 electrons. These speeds come out independent of the radius of the ring, and dependent only upon the number in the ring. The carbon atom has been selected in making this assumption, and two forms of it are given as examples, a 12 electron, and a 6 electron atom. The values of the constants do not differ greatly for the two supposed forms of carbon. By means of this an absolute scale has been determined in Figs. I and 2. It is there given for the twelve atom assumption.

7. Two secondary curves, II. and III., Fig. I, are given as the alternative values of $\Sigma_{P} a^{2}$ for some atoms. These curves have abscissæ $\mathrm{I} / 2^{4 / 3}$ and I $/ 2^{8 / 3}$ of those in Curve I. respectively. The value of $\Sigma_{P} a^{2}$ for the atoms $\mathrm{O}, \mathrm{S}, \mathrm{Cl}$ and $\mathrm{Br}$ may occur in crystals in any one of two or three forms, their weights remaining the same, but their radii changing. This change in the radius does not affect $\Sigma_{P} \beta^{2}$. Not more than two of these forms occur in the crystals considered in case of the above elements, but it is predicted that the third form will appear in some crystals not yet studied. For example, sulphur occurs in zincblende, manganblende and iron pyrites with a value corresponding to Curve II., and in galena with a value corresponding to Curve III., and no crystal gives a value corresponding to the principal Curve I. On the other hand, chlorine in $\mathrm{NaCl}, \mathrm{KCl}$, and bromine on $\mathrm{KBr}$ give values on the Curve I., and in $\mathrm{AgCl}$ and $\mathrm{AgBr}$ in Curve II., but no value in the crystals studied falls on Curve III. for these elements.

8. Curve II., Fig. 2, with the secondary Curves IV. and V. give the values of $\Sigma_{P}(a \beta)$, proportional to the total moment of momentum of the atom. These three curves have abscissæ in the ratios $I: 2^{2 / 3}: 2^{4 / 3}$ and they respresent the alternative values that an atom of sulphur, for example, may possess in different circumstances. These curves are nearly straight lines for atomic numbers above 40 , and, were it not for the alternative values in these three curves, would give good reason to suppose that the moment of momentum for each electron is nearly constant.

9. Curve III., Fig. 2, gives the average value of $\omega / c$ for each atom, from which the average frequency of revolution may be obtained. According to it, the average frequency approaches a nearly constant minimum for the heavier elements, but may be very large for the lightest element, hydrogen.

10. A proof is given to show that the curve for $\Sigma_{P} a^{2}$ in Fig. $\mathrm{I}$ is in good 
agreement with the theory that atoms may be formed in rings in a plane as in the Meyer figures, the volume of the enclosing sphere increasing by equal steps for the addition of each electron, but this offers no explanation for the two alternative values for the same atom corresponding to Curves II. and III.

I I. According to the twelve-electron-atom-assumption the radius of the outside ring of eight is determined in centimeters to be $0.792 \times 1 \mathrm{IO}^{-10}$. This is I/3Igth part of the edge of the elementary tetrahedron in the diamond. According to the six electron assumption it is $0.614 \times 10^{-10}$ $\mathrm{cm}$. The mean frequency of revolution is about $.76 \times 10^{18}$ in both examples, that is, the order of magnitude of characteristic X-ray frequencies.

I2. A knowledge of absolute values leads to a determination of the constant in the equation for the weight of an atom, from which $\Sigma_{E} \beta^{2}$ for all the electrons in the earth is found to be $21.12 \times 10^{46}$. Dividing this by the total number of electrons in the earth, which is equal to the mass of the earth in grams times the electrons per gram, a constant quantity, namely $5.984 \times 10^{27} \times 6.05 \times 10^{23}=36.2 \times 10^{50}$, the value of $\beta^{2}$ for the average electron in the earth is $.585 \times 10^{-4}$, and $\beta=0.00765$. This result leads to a speculation that the interior of the earth may be hydrogen, or, at any rate, the very lightest of the known elements. That a result, found in such a manner comes out within the range of the possible values of $\beta$ for any atoms helps to strengthen the theory that the gravitational force is proportional to $\Sigma \beta^{2}$. It is, at least, an interesting speculation because it suggests for the first time a possibility of finding the kind of elements that make up the interior of the earth and possibly other heavenly bodies.

I3. From the mechanical forces interacting between the atoms in a crystal a formula for the bulk modulus was derived in a former paper, which is here applied to several crystals. A comparison with measured values in Table III., in the few cases where measurements are known, shows that the crystals are invariably more incompressible according to calculation than they are measured to be, although the order of magnitude of the two results corresponds. It is suggested that the great variation in the measured values among different crystals shows a certain degree of interpenetration of the compressing substance and the substance compressed. This would always have the effect of reducing the apparent incompressibility, in the direction that the measurements indicate. New measurements of these quantities may well be made, employing the same crystal with different compressing substances. If different values are then obtained, the fact may be attributed to different degrees of interpenetration. Liquids, however, which have no space lattice formation may 
act very much alike. If the compressing substance were a solid it would be better.

14. The derivation of the force equations from the cubic space lattice formation is given in an appendix so as to interfere less with the continuity of the argument. A section of this is devoted to a consideration of the error that is made in neglecting the more distant atoms in the crystal than those included in a cube of edge four times the elementary cube. This error is appreciable but not excessively great, as indicated by calculating the total force due to all atoms along selected radii in the crystal to an infinite distance. The chief effect of such error is to alter somewhat the values of the constants $d, v$ and $u$ in the equilibrium conditions (5), (8) and (9) for different lattices. But, the fact that we obtain good curves in Figs. I and 2 from different crystals and different lattices goes to show that there is not a great error in these constants due to the omission of atoms more distant than those calculated.

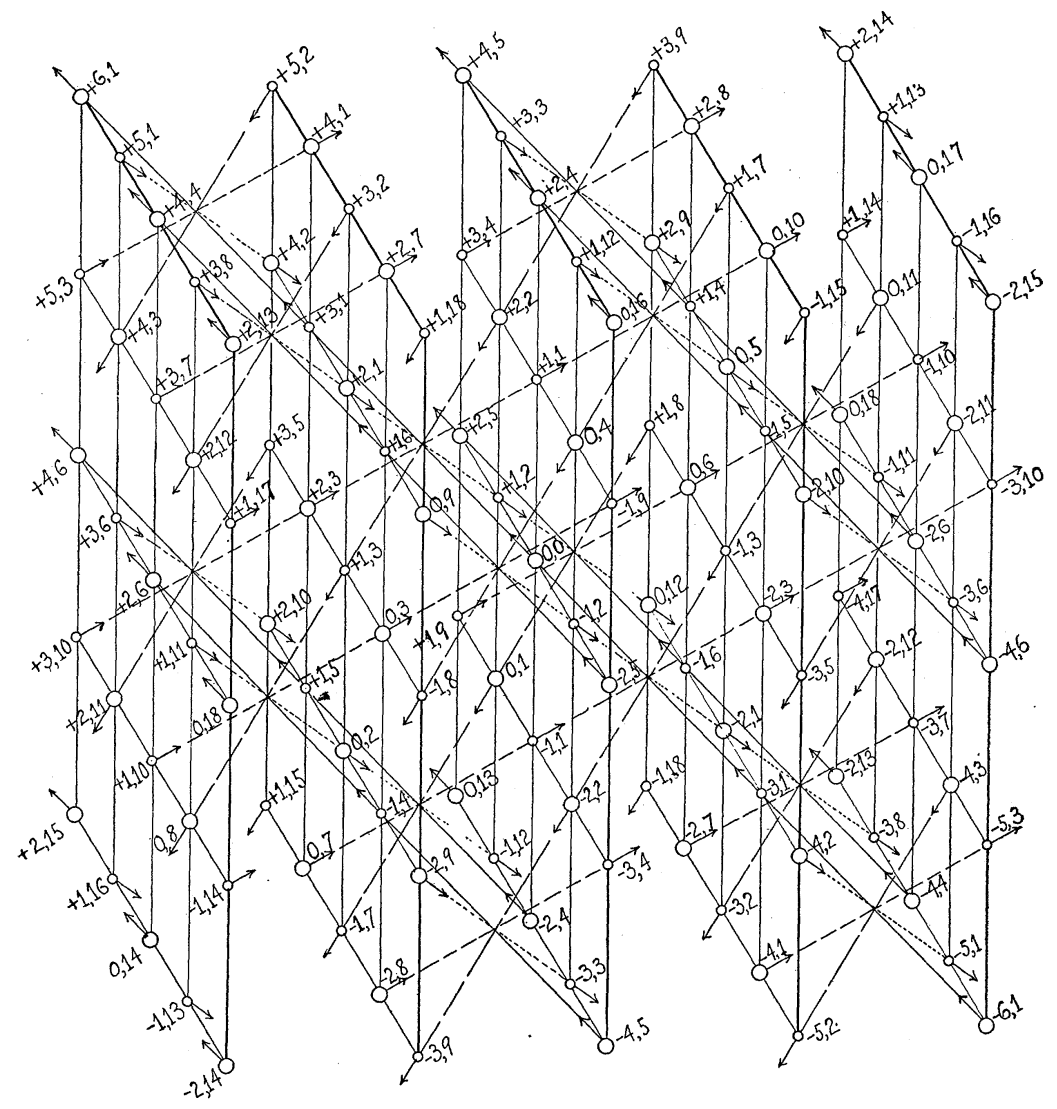

Fig. 3. 
TABLE IV.

\begin{tabular}{|c|c|c|c|c|c|c|c|c|c|c|c|c|c|c|}
\hline \multirow{2}{*}{ No } & $\dot{g}$ & \multirow{2}{*}{$x$. } & \multirow{2}{*}{$y$. } & \multirow{2}{*}{$z}$. & \multirow{2}{*}{$r_{0}$} & \multirow{2}{*}{ l. } & \multirow{2}{*}{$m}$. & \multirow{2}{*}{$n$. } & \multirow{2}{*}{ a. } & \multirow{2}{*}{$\cos a}$. & \multirow{2}{*}{$\sin \alpha$} & \multicolumn{3}{|c|}{ + Direction of Axis. } \\
\hline & No. & & & & & & & & & & & $x$. & $y$ & $z$. \\
\hline-6 & 1 & 0 & 0 & $-2 \sqrt{3} l$ & $2 \sqrt{3} l$ & 0 & 0 & -1 & 0 & +1 & 0 & - & & $0,+6-1$ \\
\hline-5 & 1 & $+\frac{1}{3} \sqrt{6} l$ & “ & $-\frac{5}{3} \sqrt{2} l$ & $3 l$ & $+\frac{1}{9} \sqrt{6}$ & “ & $-\frac{5}{9} \sqrt{3}$ & $109^{\circ} 28^{\prime} .3$ & $-\frac{1}{3}$ & $+\frac{2}{3} \sqrt{2}$ & 0,12 & 0,14 & " \\
\hline “" & 2 & “" & ". & " & " & " & $"$ & " & “ & “ & " & 0,8 & $0,16 \mid$ & " \\
\hline " & 3 & & & & " & “ & "“ & " & " & “ & " & 0,10 & 0,18 & “" \\
\hline-4 & 1 & $-\frac{1}{3} \sqrt{6} l$ & “” & $-\frac{4}{3} \sqrt{3} l$ & $\sqrt{6} l$ & $-\frac{1}{3}$ & “" & $-\frac{2}{3} \sqrt{2}$ & “ & “" & “ & " & “ & “" \\
\hline “ & 2 & “ & “ & “ & “ & “" & “ & “ & “ & " & " & 0,12 & 0,14 & “ \\
\hline “" & 3 & “ & “ & " & " & “ & “ & “ & “" & “ & “" & 0,8 & $|0,16|$ & " \\
\hline “ & 4 & $+\frac{2}{3} \sqrt{6} l$ & “ & “" & $2 \sqrt{2} l$ & $+\frac{1}{3} \sqrt{3}$ & “ & $-\frac{1}{3} \sqrt{6}$ & 0 & +1 & 0 & 0,12 & 00,14 & “ \\
\hline “" & 5 & “ & “" & “" & “" & “" & ". & “ & “" & “ & “" & 0,8 & $0,16 \mid$ & “ \\
\hline " & 6 & “ & “ & " & “ & “ & “ & “ & " & “" & “ & 0,10 & 0,18 & \\
\hline-3 & 1 & 0 & “ & $-\sqrt{3} l$ & $\sqrt{3} l$ & 0 & “ & -1 & “" & “" & “ & - & - & “ \\
\hline “ & 2 & “ & $-\sqrt{2} l$ & “ & $\sqrt{5} l$ & “ & $-\frac{1}{5} \sqrt{10}$ & $-\frac{1}{5} \sqrt{15}$ & $109^{\circ} 28^{\prime} .3$ & $-\frac{1}{3}$ & $+\frac{2}{3} \sqrt{2}$ & 0,8 & $0,16 \mid$ & “" \\
\hline “ & 3 & “ & $+"$ & “ & “ & “ & $+\cdots$ & & “ & " & “ & 0,12 & 0,14 & " \\
\hline “ & 4 & “" & $-" \cdot$ & “" & “ & “" & - " & “ & “" & " & “" & 0,10 & 0,18 & “ \\
\hline “" & 5 & " & $+"$ & “" & " & “" & $+\cdots$ & “ & “ & “ & “ & 0,8 & $0,16 \mid$ & “" \\
\hline “" & 6 & “" & - " & “" & " & “" & - $" \cdots$ & “" & " & “، & “" & 0,12 & 0,14 & " \\
\hline “ & 7 & “ & $+"$ & “ & “ & “ & $+\cdots$ & “ & “" & “ & “ & 0,10 & 00,18 & “ \\
\hline “ & 8 & $+\sqrt{6} l$ & 0 & “ & $3 l$ & $+\frac{1}{3} \sqrt{6}$ & 0 & $-\frac{1}{3} \sqrt{3}$ & " & " & " & 0,12 & 0,14 & “ \\
\hline “" & 9 & “" & "“ & "، & “" & "“ & "، & ". & “" & “" & “" & 0,8 & 0,16 & " \\
\hline & 10 & 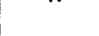 & 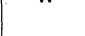 & 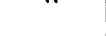 & & & & & " & " & “ & 0,10 & 0,18 & “" \\
\hline-2 & 1 & $+\frac{1}{3} \sqrt{6} l$ & “ & $-\frac{2}{3} \sqrt{3} l$ & $\sqrt{2} l$ & $+\frac{1}{3} \sqrt{3}$ & “ & $-\frac{1}{3} \sqrt{6}$ & " & " & “ & 0,12 & 0,14 & “ \\
\hline " & 2 & “ & " & "“ & “" & "“ & " & , & “" & " & “" & 0,8 & $0,16]$ & “" \\
\hline “ & 3 & $+\frac{2}{2} \sqrt{6}$ & “ & ، & 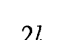 & $+\frac{1}{6}$ & “. & $-1 \sqrt{3}$ & 0 & +1 & a & 0,10 & {$\left[\begin{array}{l}0,18 \\
0\end{array}\right.$} & “" \\
\hline “ & 5 & " & “ & “" & " & $T 3 v 0$ & “" & $3 \mathrm{vo}$ & “. & "1 & " & {$\left[\begin{array}{l}1 \\
0,9\end{array}\right.$} & {$\left[\begin{array}{ll}0 & 13 \\
0 & 17\end{array}\right]$} & “ \\
\hline “ & 6 & “ & “ & “" & “ & “" & “ & “ & “ & “" & “" & 0,11 & 0,13 & “" \\
\hline " & 7 & $-\frac{2}{3} \sqrt{6} l$ & $+\sqrt{2} l$ & “ & $\sqrt{6} l$ & $-\frac{2}{3}$ & $+\frac{1}{3} \sqrt{3}$ & $-\frac{1}{3} \sqrt{2}$ & $109^{\circ} 28^{\prime} .3$ & $-\frac{1}{3}$ & $+\frac{2}{3} \sqrt{2}$ & 0,10 & 0,18 & “ \\
\hline " & 8 & “ & $-" “$ & “ & “" & "“ & $-" “$ & 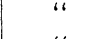 & “ & “ & “ & “ & & " \\
\hline “ & $\begin{array}{r}9 \\
10\end{array}$ & “، & I." & “ & “ & “" & $\pm \cdots$ & “" & “" & “. & “" & 0,12 & {$\left[\begin{array}{c}0,14 \\
\text { "، }\end{array}\right.$} & “" \\
\hline “" & 11 & “، & $+\cdots$ & “ & “ & “ & $+\cdots$ & " & “ & " & " & $l_{1} 8$ & 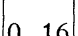 & " \\
\hline “" & 12 & “ & - “ & “ & “ & ، & $-\cdots$ & “" & ، & “ & “ & ". & {$[0,10$} & " \\
\hline-2 & 13 & $+\frac{4}{3} \sqrt{6} l$ & 0 & “ & $2 \sqrt{3} l$ & $+\frac{2}{3} \sqrt{2}$ & 0 & $-\frac{1}{3}$ & 0 & +1 & 0 & 0,12 & 0,14 & " \\
\hline " & 14 & " & " & " & " & ، & “" & 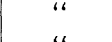 & “" & " & “" & 0,8 & $0,16 \mid$ & \\
\hline-1 & $\begin{array}{r}15 \\
1\end{array}$ & $-\frac{1}{3} \sqrt{6} l$ & “ & $-\frac{1}{3} \sqrt{3} 7$ & l & $-\frac{1}{3} \sqrt{6}$ & “" & $-\frac{1}{2} \sqrt{3}$ & $109^{\circ} 28^{\prime} .3$ & $-\frac{1}{1}$ & $+\frac{2}{3} \sqrt{2}$ & 0,10 & 0,18 & “" \\
\hline "“ & 2 & " & “ & $\begin{array}{lll}3 \\
3\end{array}$ & “، & $-3 v 0$ & “ & & " & ${ }^{3}$ & & 0,12 & 0,14 & “ \\
\hline “ & 3 & “" & “ & “" & “ & “ & “ & “" & “ & “ & “ & 0,8 & $|0,16|$ & “ \\
\hline “ & 4 & $+\frac{2}{3} \sqrt{6} l$ & “ & “ & $\sqrt{3} l$ & $+\frac{2}{3} \sqrt{2}$ & “ & $-\frac{1}{3}$ & 0 & +1 & 0 & " & T" & “ \\
\hline " & 5 & & “" & “" & & & “ & & & & “" & 0,10 & 0,18 & “ \\
\hline “ & 6 & “ & “ & “ & “ & “" & “" & “ & “" & “ & “" & 0,12 & 0,14 & “ \\
\hline “ & 7 & “ & $-\sqrt{2} l$ & " & $\sqrt{5} l$ & $+7_{15}^{2} \sqrt{30}$ & $-\frac{1}{5} \sqrt{10}$ & $-\frac{1}{15} \sqrt{15}$ & $\mid 109^{\circ} 28^{\prime} .3$ & $-\frac{1}{3}$ & $+\frac{2}{3} \sqrt{2}$ & 0,8 & $|0,16|$ & “ \\
\hline
\end{tabular}


456

ALBERT C. CREHORE.

$\left[\begin{array}{l}\text { SECOND } \\ \text { SERIES. }\end{array}\right.$

\begin{tabular}{|c|c|c|c|c|c|c|c|c|c|c|c|c|c|c|}
\hline \multirow{2}{*}{$\frac{\stackrel{\dot{g}}{\ddot{a}}}{\text { No. }}$} & \multirow{2}{*}{$\begin{array}{l}\dot{j} \\
\dot{a} \\
\end{array}$} & \multirow{2}{*}{$x}$. & \multirow{2}{*}{$y}$. & \multirow{2}{*}{$z$. } & \multirow{2}{*}{$r}$. & \multirow{2}{*}{ l. } & \multirow{2}{*}{$m}$. & \multirow{2}{*}{$n}$. & \multirow{2}{*}{$a}$. & \multirow{2}{*}{$\cos \alpha$} & \multirow{2}{*}{$\sin \alpha$} & \multicolumn{3}{|c|}{+ Direction of Axes. } \\
\hline & & & & & & & & & & & & $x$ & $y$. & $z$. \\
\hline-1 & 8 & $+\frac{2}{3} \sqrt{6} l$ & $+\sqrt{2} l$ & $-\frac{1}{3} \sqrt{3} l$ & $\sqrt{5} l$ & $+\frac{2}{15} \sqrt{30}$ & $+\frac{1}{5} \sqrt{10}$ & $-\frac{1}{15} \sqrt{15}$ & $109^{\circ} 28^{\prime} .3$ & $-\frac{1}{3}$ & $+\frac{2}{3} \sqrt{2}$ & 0,8 & 0,16 & $+6-1$ \\
\hline “" & $\begin{array}{r}9 \\
10\end{array}$ & “" & $\mid \begin{array}{l}-“ \\
+\cdots\end{array}$ & “ & “" & “" & $\begin{array}{l}- \\
\end{array}$ & “" & “ & “" & $"$ & 0,10 & 0,18 & “" \\
\hline “" & $\begin{array}{l}10 \\
11\end{array}$ & “" & $t_{-}^{+} \cdot "$ & “" & " & “" & $\left.\right|_{-} ^{+} "$ & “" & “" & " & “ & 12 & 14 & “" \\
\hline “ & 12 & " & $+"$ & " & " & “ & $+\cdots$ & “ & “ & “ & " & a, "x & on & “ \\
\hline “ & 13 & $-\frac{1}{3} \sqrt{6}$ & $+2 \sqrt{2} l$ & “ & $3 l$ & $-\frac{1}{9} \sqrt{6}$ & $+\frac{2}{3} \sqrt{2}$ & $-\frac{1}{9} \sqrt{3}$ & “" & " & " & “ & " & " \\
\hline “ & 14 & “ & $-“$ & “ & “ & “" & $-\cdots$ & & “ & “ & “ & 0,10 & 00,18 & “ \\
\hline “ & 15 & " & $+"$ & “ & “ & “ & $+\cdots$ & “ & “ & “ & “ & 0,8 & 0,16 & “ \\
\hline " & 16 & " & $-"$ " & “ & “ & “ & $-\cdots$ & “ & “ & " & " & 0,12 & 20,14 & “ \\
\hline “ & 17 & “ & $+"$ & " & “ & “ & $+\cdots$ & “ & " & " & “ & 0,10 & 00,18 & " \\
\hline “" & 18 & " & - “ & " & “ & “ & $-\cdots$ & “ & “ & ، & “ & 0.8 & 0,16 & “ \\
\hline 0 & 1 & 0 & $+\sqrt{2} l$ & 0 & $\sqrt{2} l$ & 0 & -1 & 0 & “ & “ & " & "' & " & “ \\
\hline & 2 & “ & $+"$ & “ & " & “ & $+\cdots$ & “ & “ & " & " & 0,12 & 20,14 & “ \\
\hline “ & 3 & “ & $-"$ & " & “ & “" & $-\cdots$ & “ & “ & “ & " & 0,10 & 0,18 & “ \\
\hline “ & 4 & “ & $+" “$ & “ & “ & “ & $+\cdots$ & “ & “ & " & “ & 0,8 & $0,16 \mid$ & “ \\
\hline “ & 5 & “ & _ " & “ & " & “" & - $" ،$ & " & "، & “ & “" & 0,12 & 0,14 & " \\
\hline “ & 6 & " & $+\cdots$ & “ & " & " & $+\cdots$ & " & “ & “" & " & 0,10 & 0,18 & “ \\
\hline “ & 7 & $-\sqrt{3} l$ & 0 & " & $\sqrt{6} l$ & -1 & 0 & “ & " & " & " & I" & $7 " 1$ & " \\
\hline “ & 8 & $+\cdots$ & “ & “ & “" & + & " & " & “" & " & " & 0,8 & 0,16 & " \\
\hline " & 9 & $-\cdots$ & “ & “ & " & - & “ & “ & “ & “ & “ & 0,12 & 20,14 & " \\
\hline " & 10 & $+\cdots$ & " & “" & " & $+\cdots$ & " & “" & “" & ، & "، & 0,10 & 0,18 & “ \\
\hline “ & 11 & $-\cdots$ & “ & “ & “ & - & “ & “ & “ & “" & “ & 0,8 & 0,16 & “ \\
\hline “ & 12 & $+\cdots$ & “ & “ & " & $+\cdots$ & “ & “ & “ & " & “ & 0,12 & $2|0,14|$ & “ \\
\hline “ & 13 & $+2 \sqrt{2} l$ & “ & “ & $2 \sqrt{2} l$ & a & “ & “ & 0 & +1 & 0 & 0,13 & & “ \\
\hline “ & 14 & & " & “ & & " & “ & “ & " & & " & 0,14 & 40,9 & “ \\
\hline “" & 15 & “" & “ & " & " & ، & " & ، & “" & " & “" & 0,15 & 50,10 & " \\
\hline “ & 16 & “ & " & “ & “ & “ & “ & “ & “ & “ & “ & & 60,11 & “ \\
\hline “ & 17 & “ & “ & " & “ & “ & “ & “ & “" & “ & “ & 0,17 & 70,12 & “ \\
\hline " & 18 & " & “ & “ & " & “ & “ & “ & " & “ & “ & 0,18 & 80,7 & “ \\
\hline
\end{tabular}

Appendix.

In the case of the diamond lattice the coördinates of position of the surrounding atoms, with respect to a selected atom at the origin of the coördinates, were given ${ }^{1}$ in Table I., page 264 . In a similar manner the following Table IV. gives them for a simple cubic lattice like that of rock salt. The atoms are numbered as in the diagram Fig. 3.

The calculation of the numerical values of the functions $f_{4}$ and $f_{6}$, given in equations (I) and (2) above, has been carried out for each of the I24

${ }^{1}$ Loc. cit. 
atoms surrounding the central atom in a cube of edge $4 l$, giving the results in Table V. Each atom in the $(x, y)$ or $z=0$, plane gives $F_{z}=0$ because $n$ is a factor of (I) for these atoms, and is zero in the plane $z=0$.

\section{TABLE V.}

Odd Planes.

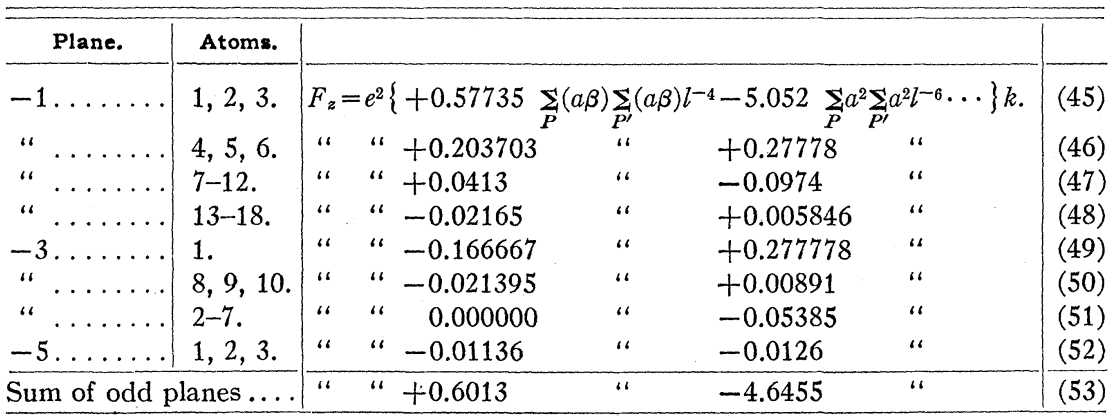

Even Planes.

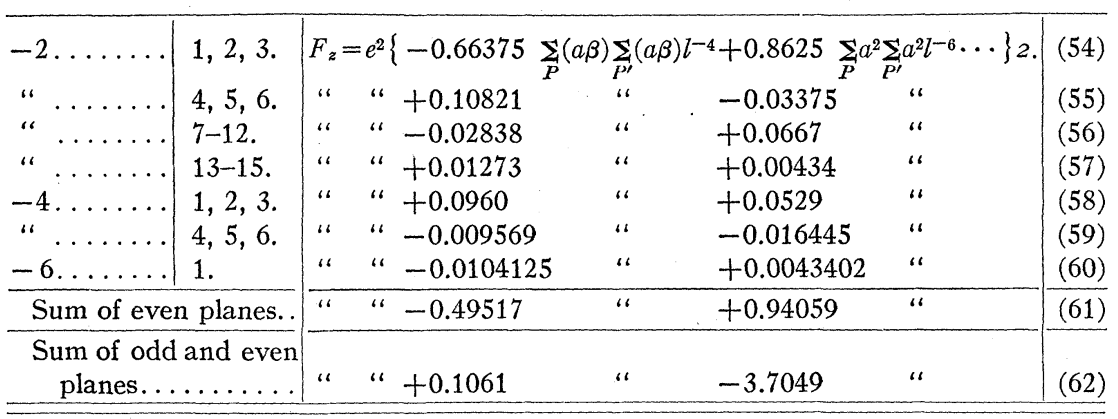

The forces in the Table $\mathrm{V}$. are given for the negative planes only, those on one side of the central atom, thus including but one half of the cube of edge $4 l$. In this form of lattice it happens that each atom is a center of symmetry, not only for the position and kind of atom, but for the direction of its axis of rotation as well. It always happens that there is an atom of chlorine in rock salt, for example, having its axis parallel to a corresponding atom of chlorine at an equal distance on the opposite side of the selected atom. Equation (I) shows that the force that one pair of such atoms exerts upon the selected atom is zero irrespective of the value of $l$. The force changes sign if we reverse the signs of $l, m$ and $n$, the direction cosines, $a$ remaining the same. The size of the space lattice is, therefore, indeterminate if we consider the whole cube. This makes it possible to impose a second condition before the size of the lattice is fixed. This is, that we must obtain the same value of $l$ whether we 
select a sodium or a chlorine atom upon which to figure the force. We get the same value of $l$ by equating to zero the total force of all the sodium atoms in one half the cube, acting upon a selected chlorine atom, or by equating to zero the total force of all the chlorine atoms in one half the cube acting upon a selected sodium atom. Since the odd planes always contain atoms of the opposite kind from the selected atom, it is concluded that the equilibrium condition for all crystals like rock salt is obtained by equating (53), which is the same as (6) to zero.

\section{Degree of Approximation.}

Had we included in the calculation the innermost cube only, of edge $2 l$, we should have had for the odd planes only the forces given in (45), (46) and (49), and for the even planes only (54), giving the results

Odd planes, $\quad F_{z}=e^{2}\left\{+0.6144 \sum_{P}(a \beta) \sum_{P^{\prime}}(a \beta) l^{-4}\right.$

$$
\left.-4.4964 \underset{P}{\Sigma_{P}} a_{P^{\prime}}^{2} a^{2} l^{-6} \cdots\right\} k, \quad(63)
$$

Even planes, $F_{z}=e^{2}\left\{-0.66375 \underset{P}{\Sigma_{P}}(a \beta) \underset{P^{\prime}}{\Sigma_{1}}(a \beta) l^{-4}\right.$

$$
\left.+0.8625 \sum_{P} a^{2} \sum_{P^{\prime}} a^{2} l^{-6} \cdots\right\} k
$$

which may be compared directly with (53) and (6I) above. The inclusion of the next outlying cube of atoms makes less difference here in the case of the odd planes than in that of the even. But, the question arises how much the results in (53) and (6I), which have been used in the paper, would be changed if we had included more of the outlying atoms. This question can not be answered exactly until such calculations are made. They involve considerable labor and have not been carried out beyond the cube of edge $4 l$, so that it is very desirable to obtain some kind of an estimate of the error made in neglecting the more distant atoms. To help give some idea of this error the forces due to all the atoms lying along certain radii to infinity have been obtained in the case of the even planes (6I), which showed the greater difference when the outer cube was included.

For example, along the radius vector from the origin to atom $(-6, \mathrm{I})$ we come to atoms of the same kind and with axes parallel to the atom at the origin at equal intervals, the second being twice, and third three times as far, and so on, as atom $(-6,1)$, the force of which is given by (60). Since the first term varies as $r^{-4}$, we may use this equation to find the force of all other atoms along this radius to infinity. This is equivalent to multiplying (6o) by the sum of the series to infinity 


$$
2^{-4}+3^{-4}+4^{-4}+5^{-4} \cdots=0.082323,^{1}
$$

to obtain the coefficient of the fourth power term due to all other outlying atoms along this radius. Similarly, for the sixth power term, we have to sum the series

$$
2^{-6}+3^{-6}+4^{-6}+5^{-6} \cdots=0.017343 .
$$

This result would have made equation (6o), if we include all atoms along this radius to infinity, have the coefficient of $l^{-4}-0.0008565$, and of $l^{-6} 0.0000755$, different from that given in (6o).

In a similar manner all atoms along several different radii have been included, giving the following amounts to be added to the coefficients as given in the Table $\mathrm{V}$.

\begin{tabular}{c|c|c}
\hline \multicolumn{1}{c|}{ Direction of Radius. } & Coef. of $l^{-4}$. & \multicolumn{1}{c}{ Coef. of $l^{-6}}$. \\
\hline$(-6,1) \ldots \ldots \ldots \ldots \ldots \ldots \ldots \ldots \ldots$ & -0.0008565 & 0.0000755 \\
$(-2,4)(-2,5)(-2,6) \ldots \ldots \ldots$ & 0.008903 & 0.0005853 \\
$(-4,4)(-4,5)(-4,6) \ldots \ldots \ldots$ & 0.0007875 & 0.0002852 \\
$(-2,1)(-2,2)(-2,3) \ldots \ldots \ldots$ & 0.00969 & 0.001240 \\
$(-4,1)(-4,2)(-4,3) \ldots \ldots \ldots$ & 0.001425 & 0.00007606 \\
\hline Sum. $\ldots \ldots \ldots \ldots \ldots \ldots \ldots$ & 0.001006 & 0.00052106 \\
\hline
\end{tabular}

When these values are added to the coefficients in $(6 \mathrm{I})$, it changes the fourth power coefficient by about one part in five hundred, and the sixth power by about one part in two thousand. . The atoms added by this process do not include all, even in the next adjacent cube with edge $6 l$, but these figures indicate that we can not rely upon the accuracy of the figures in the Table $\mathrm{V}$. in the third decimal place in the fourth power coefficient, and it is not now possible to say whether the value will turn out to be larger or smaller than those in (6I), if we were to include all surrounding atoms to a great distance. The preceding, however, may be regarded as showing something as to the order of the error made by omitting outlying atoms at greater distances.

\section{The Diamond Lattice.}

The diamond form of lattice consists of two interpenetrating facecentered lattices. If we number the (III) planes consecutively, from o for the selected atom as before, the even planes in one of the lattices are symmetrical with respect to the selected atom, giving a total force of zero irrespective of the size of the lattice. The odd planes are not symmetrical with respect to it, and the force must be calculated for all odd planes, both positive and negative since these do not cancel each

1 For a table of the sums of these series, see De Morgan's Calculus, p. $55^{2}$. 
other. In the case of $\mathrm{ZnS}$ the planes alternate, even planes being zinc and odd sulphur, if we select a zinc atom on which to figure the force. The equilibrium condition must be the same whether we select a zinc or a sulphur atom upon which to figure the force as in the case of rock salt.

The crystal $\mathrm{CaF}_{2}$ has a structure similar to $\mathrm{ZnS}$ in some respects. If $\mathrm{Ca}$ is substituted for $\mathrm{Zn}$ and one of the F's for $\mathrm{S}$, the arrangement so far is the same. The $\mathrm{Ca}$ planes recur at regular intervals, this interval being the altitude of the tetrahedron on the elementary triangular base in the Ca plane. One of the $\mathrm{F}$ planes occurs at one quarter of this distance above the $\mathrm{Ca}$ plane, similar to the $\mathrm{S}$ in $\mathrm{ZnS}$, and the other $\mathrm{F}$ at the same distance below the $\mathrm{Ca}$ plane, which has no corresponding plane in $\mathrm{ZnS}$. The spacing of the planes then gives the following regular succession

F-Ca-F-Space-F-Ca-F-Space-F-Ca-F-Space and so on,

there being three planes at regular intervals and then one missing. From this it is evident that if we select a $\mathrm{Ca}$ atom upon which to figure the force, the positive planes will just balance the negative planes, thus making the size of the lattice indeterminate. For the equilibrium condition, therefore, we do not need to figure the force upon a calcium atom. The case is different if we select one of the F atoms, for, then the Ca atoms are not symmetrical about it. On the other hand, the F atoms are symmetrical about it. This narrows the condition down to precisely the same formula as applies to the diamond or zincblende, because we only have to take into account the force of all the $\mathrm{Ca}$ atoms upon a fluorine atom. 